\title{
Glycinergic Pacemaker Neurons in PreBötzinger Complex of Neonatal Mouse
}

\author{
Consuelo Morgado-Valle, ${ }^{1,2}$ Serapio M. Baca, ${ }^{1}$ and Jack L. Feldman ${ }^{1}$ \\ ${ }^{1}$ Systems Neurobiology Laboratory, Department of Neurobiology, David Geffen School of Medicine at UCLA, University of California, Los Angeles, \\ Los Angeles, California 90095-1763, and 2Programa de Neurobiologia, Universidad Veracruzana, Xalapa, Veracruz, Mexico, C.P. 91190
}

The preBötzinger complex (preBötC) is essential for normal respiratory rhythm generation in rodents, for which the underlying mechanisms remain unknown. Excitatory preBötC pacemaker neurons are proposed to be necessary for rhythm generation. Here we report the presence of a population of preBötC glycinergic pacemaker neurons. We used rhythmic in vitro transverse slice preparations from transgenic mice where neurons expressing the glycine transporter 2 (GlyT2) gene coexpress enhanced green fluorescent protein (EGFP). We combined epifluorescence and whole-cell patch-clamp recording to study preBötC EGFP-labeled, i.e., glycinergic, inspiratorymodulated neurons with pacemaker properties. We defined glycinergic pacemaker neurons as those preBötC EGFP neurons that exhibited the following: (1) ectopic bursting in rhythmic slices when depolarized during their normally silent period and (2) bursting when depolarized in nonrhythmic slices (following AMPA receptor blockade). Forty-two percent of EGFP-labeled neurons were inspiratory ( $n=48$ of 115$)$, of which $23 \%$ ( $n=11$ of 48 inspiratory; $10 \%$ of the total recorded) were pacemakers. We conclude that there is a population of preBötC inspiratory-modulated glycinergic, presumably inhibitory, pacemaker neurons that constitute a substantial fraction of all preBötC pacemaker neurons. These findings challenge contemporary models for respiratory rhythmogenesis that assume the excitatory nature of preBötC pacemaker neurons. Testable and nontrivial predictions of the functional role of excitatory and inhibitory pacemaker neurons need to be proposed and the necessary experiments performed.

\section{Introduction}

The preBötzinger complex (preBötC) is essential for normal breathing in rodents (Tan et al., 2008) and is postulated to be an essential site for respiratory rhythm generation (Smith et al., 1991). In brainstem-spinal cord (en bloc) and transverse slice in vitro preparations, respiratory rhythm persists in the absence of postsynaptic inhibition (Feldman and Smith, 1989; Onimaru et al., 1990; Shao and Feldman, 1997; Brockhaus and Ballanyi, 1998). This observation led to the hypothesis that intrinsically rhythmic excitatory pacemaker neurons drive the respiratory rhythm (Smith et al., 1991); preBötC neurons with pacemaker properties dependent on persistent sodium current $\left(I_{\mathrm{NaP}}\right)$ or $\mathrm{Ca}^{2+}$-activated nonspecific cationic current $\left(I_{\mathrm{CAN}}\right)$ of undetermined neurotransmitter phenotype were subsequently identified (Johnson et al., 1994; Thoby-Brisson and Ramirez, 2001; Del Negro et al., 2002; Peña et al., 2004). Many models for rhythmogenesis predicate a significant role, often obligatory, for excitatory, presumably glutamatergic, pacemaker neurons (Butera et al., 1999a,b; Smith et al., 2000; Del Negro et al., 2001; Rybak et al.,

Received June 26, 2009; revised Nov. 3, 2009; accepted Dec. 16, 2009.

This research was supported by National Institutes of Health Grants R01 HL-040959 and F32-HL087589. C.M.-V. is a Parker B. Francis fellow in Pulmonary Research (Francis Family Foundation, Kansas City, M0). We thank Dr. S. du Lac for providing GlyT2-EGFP mice, which are derived from Dr.Zeilhofer's GlyT2-EGFP lines; Dr. M. Bagnall for sharing preliminary data and helpful discussions throughout; and M. Fuentes and G. Li for technical assistance.

Correspondence should be addressed to Dr. Consuelo Morgado-Valle, Programa de Neurobiologia, Universidad Veracruzana, Calle de la Pedreta No. 55 Pedregal de las Animas, Xalapa, Veracruz, Mexico, C.P. 91190. E-mail: comorgado@uv.mx.

D0I:10.1523/JNEUROSCI.3040-09.2010

Copyright $\odot 2010$ the authors $\quad 0270-6474 / 10 / 303634-06 \$ 15.00 / 0$
2003, 2008). Pharmacological studies suggest that pacemaker neurons are not obligatory for rhythmogenesis (Del Negro et al., 2002, 2005; Feldman and Del Negro, 2006). Regardless, they could play a role in modulating/stabilizing the rhythm (Purvis et al., 2007). Recently, preBötC pacemakers expressing the vesicular glutamate transporter 2 (VGluT2) and presumably glutamatergic were identified, but they were few in number and may not possess pacemaking properties under normal conditions (St-John et al., 2009).

The preBötC contains excitatory and inhibitory neurons (Stornetta et al., 2003; Winter et al., 2009). How pacemakers are distributed between these two populations is unknown. PreBötC rhythm is abolished in vitro (Greer et al., 1991) and in vivo (Chitravanshi and Sapru, 1996) after blockade of glutamatergic transmission. Although inhibition is not necessary for rhythmogenesis in the preBötC in in vitro slices (Feldman and Smith, 1989; Del Negro et al., 2009), inhibition is important in respiratory patterning (Feldman and Smith, 1989; Bianchi et al., 1995; Shao and Feldman, 1997; Brockhaus and Ballanyi, 1998; Büsselberg et al., 2001; Richter and Spyer, 2001; Ren and Greer, 2006). In some invertebrates, inhibitory pacemakers play a fundamental role in rhythmic behavior (Cardi and Nagy, 1994; Mamiya and Nadim, 2004). Here, we sought to determine whether there are inhibitory neurons with pacemaker properties within the preBötC. We recorded from inspiratory-modulated glycinergic neurons in an in vitro transverse slice preparation from mice with EGFP expressed in GlyT2-containing neurons (Zeilhofer et al., 2005). We used whole-cell recording and epifluorescence to test for two distinct pacemaker properties: (1) ectopic 

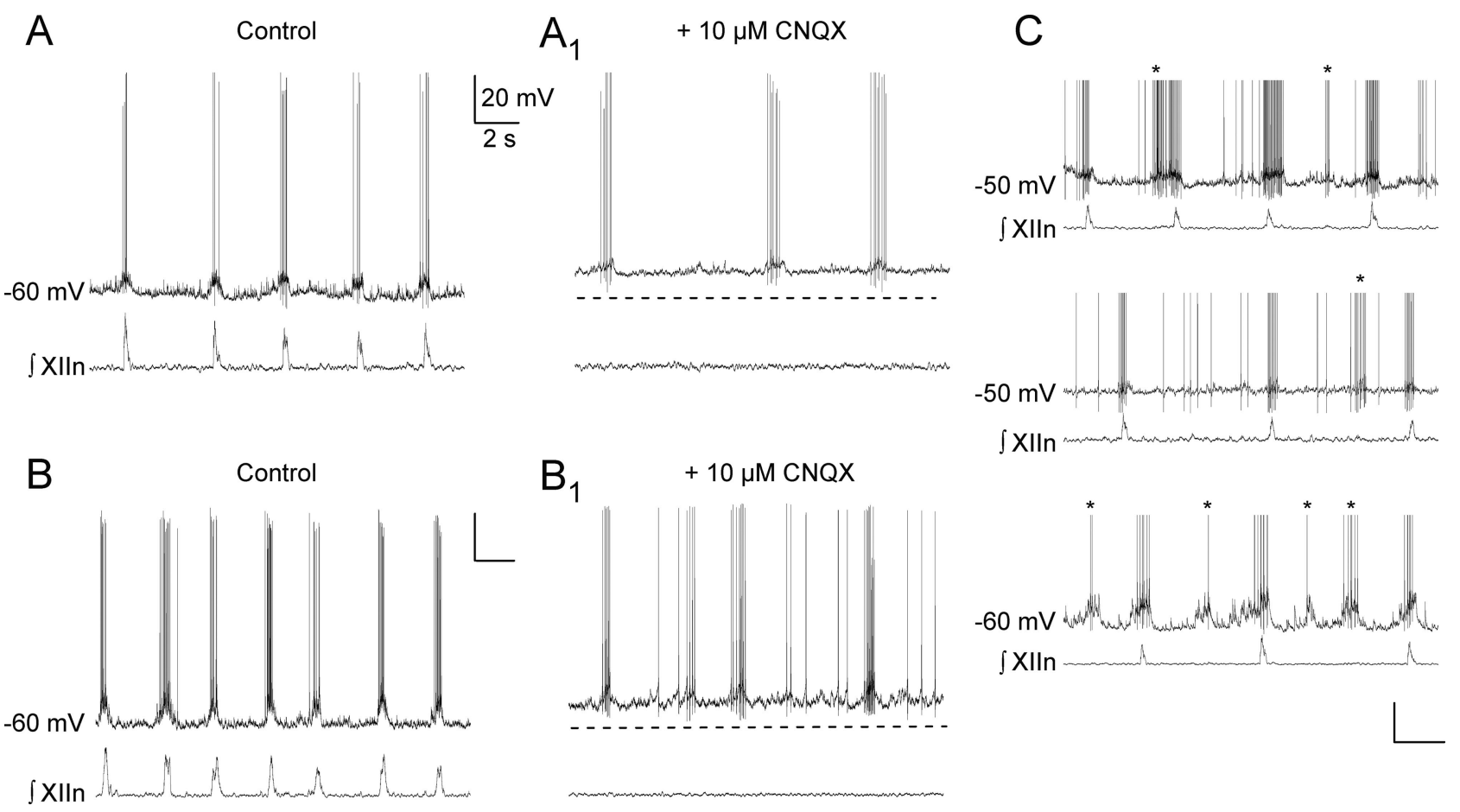

Figure 1. Examples of preBötC GlyT2-EGFP pacemaker neurons. $A$, Respiratory-modulated discharge in a GlyT2-EGFP pacemaker neuron current clamped at interburst $V_{\mathrm{m}} \approx-60 \mathrm{mV}$. A1, Bursting activity in $10 \mu \mathrm{m}$ CNQX bath applied. Network activity is blocked. B, B1, Another example of a respiratory-modulated discharge and bursting in a GlyT2-EGFP neuron. C, Examples of respiratory-modulated discharge and "ectopic bursts" (asterisks) after depolarizing $V_{\mathrm{m}}$. Action potentials have been truncated.

bursting induced by depolarization during their silent period in rhythmic slices and (2) bursting induced by depolarization in nonrhythmic slices (following AMPA receptor blockade). Approximately $23 \%$ of these inspiratory-modulated glycinergic neurons had both pacemaker properties. We conclude that the presumption that all preBötC pacemaker neurons are excitatory is incorrect. Establishing the neurotransmitter(s) used by preBötC pacemaker neurons is essential for understanding their functional role, if any, in generating or modulating respiratory pattern, and a prerequisite for validating models that stipulate pacemakers as an essential element of the rhythm generating mechanism. The inspiratory glycinergic, presumably inhibitory, pacemaker neurons in preBötC are a novel class of neurons that may modulate the respiratory network.

\section{Materials and Methods}

Medullary slice preparation. Experiments were performed on transverse brainstem slices generating respiratory-related motor output (Smith et al., 1991) from GlyT2-EGFP mice (Zeilhofer et al., 2005). The Office for the Protection of Research Subjects, University of California Research Committee approved all protocols. Mice ( $n=26, \mathrm{P} 0-\mathrm{P} 7$ from 10 litters) were anesthetized with isoflurane and decerebrated, and the neuraxis was isolated. The brainstem was serially sectioned (Vibratome) in the transverse plane until the nucleus ambiguus and inferior olive were visible. A slice $(450-500 \mu \mathrm{m})$ containing the preBötC was cut (Del Negro et al., 2002; Ruangkittisakul et al., 2006). The dissection was performed in artificial CSF (ACSF) containing (in mM) $128 \mathrm{NaCl}, 3 \mathrm{KCl}, 1.5 \mathrm{CaCl}_{2}, 1$ $\mathrm{MgSO}_{4}, 23.5 \mathrm{NaHCO}_{3}, 0.5 \mathrm{NaH}_{2} \mathrm{PO}_{4}$, and 30 glucose, bubbled with $95 \%$ $\mathrm{O}_{2} / 5 \% \mathrm{CO}_{2}$ at $27^{\circ} \mathrm{C}$. The slice was perfused with $\mathrm{ACSF}(6 \mathrm{ml} / \mathrm{min})$ in a 1 $\mathrm{ml}$ recording chamber.

Electrophysiological recording. Respiratory-related motor output was recorded from hypoglossal nerves (XIIn) using suction electrodes. To obtain a robust, stable rhythm, $\mathrm{ACSF} \mathrm{K}^{+}$concentration was elevated to 9 $\mathrm{mM}$. Slices were perfused for $30 \mathrm{~min}$ before experimental manipulations. XIIn activity was amplified, bandpass filtered $(0.3-1 \mathrm{kHz})$, rectified, and integrated ( $\left.\tau=20 \mathrm{~ms} ; \int \mathrm{XIIn}\right)$. Whole-cell patch-clamp recordings were performed using an Axopatch 200A amplifier (Molecular Devices) in current-clamp mode. preBötC inspiratory neurons were visualized using infrared-enhanced differential interference contrast (IR-DIC) video microscopy. Electrodes were pulled from borosilicate glass (outer diameter, $1.5 \mathrm{~mm}$; inner diameter, $0.86 \mathrm{~mm}$ ) and filled with solution containing the following (in mM): $130 \mathrm{~K}$-gluconate, $10 \mathrm{NaCl}, 10 \mathrm{HEPES}, 0.1 \mathrm{CaCl}_{2}, 1.1$ EGTA, 2 Mg-ATP, and 0.3 GTP-Na, pH 7.3; in some experiments, $0.01 \%$ rhodamine was added. Electrophysiological signals were low-pass filtered and digitized at $4 \mathrm{kHz}$ using pCLAMP software and a Digidata 1320 AD/DA board (Molecular Devices).

Neuron visualization. We detected EGFP-labeled neurons using an upright microscope (DMLFS, Leica) equipped for epifluorescence, a $63 \times$ objective (HCX/APO 0.90 numerical aperture, Leica), and dichroics (I3 and N2.1, Leica). In some experiments, 15-30 images were acquired with a CCD camera (Watec), digitized (Scion LG-3), and averaged (Scion Image). Image processing was performed in ImageJ (National Institutes of Health).

Drugs. Drugs were bath applied. 6-Cyano-7-nitroquinoxaline-2,3dione (CNQX, $10 \mu \mathrm{M}$ ) and flufenamic acid (FFA, 10-500 $\mu \mathrm{M})$ were obtained from Sigma Chemical. Riluzole $(10-20 \mu \mathrm{M})$ was obtained from Tocris Bioscience.

Immunohistochemistry. Mice ( $n=6, \mathrm{P} 4-\mathrm{P} 21$ from 3 litters) were anesthetized and transcardially perfused with $4 \%$ paraformaldehyde in PBS (PFA/PBS). The brainstems were dissected and placed in 4\% PFA/PBS overnight, cryoprotected in 30\% sucrose/PBS, embedded in OCT medium, and cut in $40 \mu \mathrm{m}$ transverse sections with a freezing microtome. Freely floating sections were processed for neurokinin-1 receptor (NK1R) immunolabeling (Gray et al., 1999). The preBötC was identified in transverse sections as the NK1R-immunoreactive (ir) zone ventral to the subcompact region of the nucleus ambiguus (Gray et al., 2001),

Imaging. Confocal image stacks were acquired with a Zeiss LSM 510 microscope and software. Lasers (488 and $543 \mathrm{~nm}$ ) and appropriate filters were used to visualize EGFP and NK1R-ir. To avoid cross talk between channels, and therefore false colocalization of EGFP and NK1R-ir signals, we acquired images in multitrack mode or ensured that the NK1R-ir signal was unaltered after modifications of the power of the 488 $\mathrm{nm}$ laser. We used $40 \times$ and $63 \times$ objectives to estimate soma size and determine colocalization of the EGFP and NK1R-ir signals. 
No striking qualitative differences between the shapes of EGFP-labeled and NK1R-ir somas were found. Somas were approximately spherical. Therefore we measured the diameter at the largest cross-sectional region of the neuron using confocal stacks. We did not explore differences in their neuropil nor attempt other measures. Data are expressed as the mean \pm SEM. A $t$ test was used to determine statistical differences between mean values.

\section{Results}

\section{Glycinergic inspiratory neurons}

Under epifluorescence microscopy and regardless of size or shape, EGFP-labeled neurons $(n=115)$ from P0-P7 GlyT2EGFP neonatal mice were whole-cell patch clamped at their somas. Using current-clamp mode, we maintained neuronal $V_{\mathrm{m}}$ at approximately $-60 \mathrm{mV}$ during the period between XIIn bursts. Fifty-eight percent $(n=67 / 115)$ of these neurons were either silent or had an irregular firing pattern, i.e., were nonrespiratory-modulated, and 42\% $(n=48$ / 115 ) had inspiratory-modulated membrane depolarization and spiking. Of these inspiratory-modulated neurons, 27\% (13/48) showed delayed excitation when depolarized by a square pulse from a hyperpolarized $(-70 \mathrm{mV})$ membrane potential, a signature of type 1 neurons (Rekling et al., 1996; Gray et al., 1999), and the remaining 73\% (35/48) showed a sag during a hyperpolarizing square pulse and postinhibitory rebound, a signature of type 2 neurons.

\section{Pacemaker properties in}

\section{EGFP-labeled neurons}

In rhythmic slices, when EGFP-labeled inspiratory neurons $(n=48)$ were depolarized by current injection, $37 \%(n=18 / 48)$ produced ectopic bursts of action potentials, i.e., burst out of phase with XIIn inspiratory bursts (Fig. 1C). For these latter neurons, rhythm in the slice was abolished by bath application of CNQX $(10 \mu \mathrm{M})$, blocking fast glutamatergic transmission; upon depolarization, $61 \%(n=11 / 18)$ showed voltage-dependent intrinsic bursting (Fig. $1 A, B)$.

To determine the presence of $I_{\mathrm{NaP}}$ or $I_{\mathrm{CAN}}$, we bath applied the $I_{\mathrm{CAN}}$ blocker FFA or the $I_{\mathrm{NaP}}$ blocker riluzole. EGFP-labeled preBötC pacemaker neurons were sensitive to $10 \mu \mathrm{M}$ riluzole, which abolished bursting within $3 \mathrm{~min}(n=7 / 7$; included in this group are two neurons that were insensitive to $10 \mu \mathrm{M}$ FFA, but further application of $10 \mu \mathrm{M}$ riluzole abolished intrinsic bursting). In a small sample, EGFP-labeled inspiratory preBötC pacemaker neurons were also extremely sensitive to FFA, which rapidly abolished bursting at $500 \mu \mathrm{M}(n=2 / 2$ tested $)$ or $100 \mu \mathrm{M}(n=2 / 2$ tested).

There is an early postnatal age dependence of $I_{\mathrm{CAN}}$-dependent pacemaker activity (Peña et al., 2004; Del Negro et al., 2005). Our small dataset suggests a developmental dependence of glycinergic pacemakers. We infrequently found EGFP-labeled inspiratory preBötC pacemaker neurons in transverse slices from $\mathrm{P} 0-\mathrm{P} 3$ mice ( $n=3$ pacemaker neurons from 11 slices). The likelihood of finding EGFP-labeled pacemaker neurons tripled in P4-P7 mice ( $n=8$ neurons from 10 slices).

\section{NK1R expression in EGFP-labeled inspiratory neurons}

The preBötC contains a high density of NK1R-ir neurons (Gray et al., 1999; Wang et al., 2001; Pagliardini et al., 2005). Less than $1 \%(1 / 140)$ of preBötC EGFP-labeled neurons were NK1R-ir $(n=140)$ (Fig. $2 B)$.

We measured soma size of preBötC NK1R-ir $(n=24)$ and EGFP-labeled ( $n=45$ ) neurons from three preparations. Somas of EGFP-labeled neurons were smaller than those of NK1R-ir neurons (soma diameters: EGFP-labeled $13.6 \pm 0.5 \mu \mathrm{m}$; NK1R-ir $16.3 \pm 0.6 \mu \mathrm{m} ; p<0.001)$.

\section{Discussion}

Our principal result is that in the preBötC of neonatal rodents, there is a population of pacemaker neurons that are glycinergic and inspiratory-modulated.

\section{Are the GlyT2-EGFP neurons exclusively glycinergic?}

Studies of the anatomy of GlyT2-EGFP mice using immunohistochemistry against glycine or GlyT2 showed that $>90 \%$ of EGFP 
Table 1. Estimated numbers of preBötC neurons in various categories

\begin{tabular}{|c|c|c|c|c|c|c|c|c|}
\hline \multirow[b]{2}{*}{ Neurons } & \multicolumn{2}{|c|}{ Estimated } & \multicolumn{6}{|c|}{ Recorded } \\
\hline & \# Total & $\%$ Total & \# & $\%$ of GlyT2 & $\#$ & \% GlyT2 inspiratory & \# & $\%$ Ectopic bursters \\
\hline PreBötC & 3000 & 100 & & & & & & \\
\hline${\mathrm{NK} 1 \mathrm{R}^{+}}^{+}$ & 300 & 10 & & & & & & \\
\hline GlyT2 $^{+}$ & 600 & 20 & 115 & 100 & & & & \\
\hline GlyT2 $^{+}$noninspiratory & 348 & 12 & 67 & 58 & & & & \\
\hline $\mathrm{GlyT}^{+}$inspiratory & 252 & 8 & 48 & 42 & 48 & 100 & & \\
\hline $\mathrm{GlyT}^{+}{ }^{+}$inspiratory ectopic burster & 93 & 3 & & & 18 & 37 & 18 & 100 \\
\hline $\mathrm{GlyT}^{+}{ }^{+}$inspiratory ectopic burster pacemaker & 57 & 2 & & & & & 11 & 61 \\
\hline
\end{tabular}

PreBötC, NK1R ${ }^{+}$, and GlyT2 ${ }^{+}$were estimated from histological counts. Other estimated numbers/percentages were extrapolated from recordings of neurons of various types.

fluorescent neurons are glycine-ir (in soma and dendrites) and GlyT2-ir (in axon terminals) (Zeilhofer et al., 2005).

Precursors of GABAergic interneurons in the molecular layer of the cerebellum transiently express GlyT2 during development (Simat et al., 2007). This suggests that, at least in cerebellum, GlyT2 is a marker of immature GABAergic neurons. While $\mathrm{Cl}^{-}$ currents are outward in some neurons at early stages of development, activation of glycinergic and $\mathrm{GABA}_{\mathrm{A} / \mathrm{B}}$ receptors in the mouse respiratory network in vitro in all stages of postnatal development hyperpolarizes inspiratory neurons (Ramirez et al., 1996; Zhang et al., 1999), confirming the inhibitory nature of glycinergic and GABAergic neurons. Moreover, glycine elicits IPSPs in preBötC neurons (Shao and Feldman, 1997). We are unaware of experimental evidence suggesting a dual excitatory/ inhibitory neuronal phenotype, such as the glutamatergic/ GABAergic phenotype expressed by developing hippocampal granule cells (Gutiérrez and Heinemann, 2006). While glycine modulates excitability by acting as coagonist of NMDA receptor, NMDA receptors are not required for generation of respiratory rhythm or motor output (Morgado-Valle and Feldman, 2007) in standard in vitro conditions in the medullary slice.

Here we established the presence of pacemaker properties in EGFP-labeled inspiratory preBötC neurons in GlyT2-EGFP mice. Given the lack of studies of preBötC neurons in GlyT2EGFP mice that suggest otherwise, we refer to EGFP-labeled neurons in these mice as "glycinergic" with presumptive inhibitory function.

The presence of inspiratory-modulated glycinergic, presumably inhibitory, pacemaker neurons in the preBötC constitutes a novel subpopulation of preBötC neurons that challenges the current models of respiratory rhythm generation where only excitatory pacemakers are stipulated [e.g., Butera et al. (1999a,b), Smith et al. (2000), Del Negro et al. (2001), and Rybak et al. $(2003,2008)]$. This stipulation is based on previous recordings of pacemaker neurons in the preBötC, where the nature of neurotransmission (excitatory or inhibitory) was assumed but not determined. The glycinergic pacemakers we identified cannot directly generate inspiratory rhythm in these models, because they would inhibit activity during the inspiratory phase. Recently identified excitatory pacemakers in the preBötC also appear inconsistent with these models insofar as these neurons may not possess pacemaker properties under normal conditions (St-John et al., 2009).

\section{Glycinergic neurons are not NK1R-ir}

In the preBötC, glycinergic and NK1R-ir neurons appear to be two distinct, non-overlapping populations, as we found colocalization of NK1R-ir in $\leq 1 \%$ of EGFP-labeled preBötC neurons. This lack of overlap follows from previous work. mRNA encoding GlyT2 is detected only in $~ 1 \%$ NK1R-ir neurons (Wang et al.,
2001). Furthermore, in the ventral respiratory group (VRG) at least $77 \pm 9 \%$ of NK1R-ir neurons are excitatory, as they contain mRNA that encodes the VGluT2, a reliable marker of glutamatergic neurons (Guyenet et al., 2002).

\section{Can we estimate what fraction of preBötC neurons are GlyT2-EGFP pacemakers?}

The rat preBötC contains $\sim 300$ NK1R-ir neurons that represent $\sim 10 \%$ of all preBötC neurons (Gray et al., 1999; Wang et al., 2001 ). In our experience $\sim 60 \%$ of neurons that we record in active slices from neonatal rodents are inspiratory modulated. Based on our cell counts in the mice studied here, we estimate that $\sim 20 \%$ of all preBötC neurons were EGFP labeled, making GlyT2-EGFP pacemaker neurons $\sim 2 \%$ of all preBötC neurons and $\sim 3 \%$ of preBötC inspiratory neurons (see Table 1 ). The estimate of the prevalence of pacemaker neurons in randomly recorded preBötC inspiratory neurons (with the presumption of no sampling bias, see below) ranges from 5\% (Del Negro et al., 2002 ) to $25 \%$ (Peña et al., 2004). Based on our data and estimates, GlyT2-EGFP pacemaker neurons represent $\sim 3 \%$ of preBötC inspiratory neurons, making them up to $\sim 50 \%$ of preBötC pacemaker neurons in our experimental conditions or as low as $\sim 10 \%$ in different conditions (Peña et al., 2004). Conversely, from $\sim 50 \%$ up to $\sim 90 \%$ of preBötC inspiratory pacemaker neurons may be excitatory.

A recent blind-patch study in perfused in situ preparations of juvenile (P14-P21) and neonatal (P6-P8) rats identified four preBötC intrinsic pacemaker neurons (St-John et al., 2009); three were positive for VGluT2, suggesting that they were excitatory. The remaining VGluT2-positive pacemakers (15/19) were located caudal to the preBötC in a region that does not appear obligatory for rhythmogenesis (Smith et al., 1991). Many of these VGluT2-positive neurons exhibited pacemaker properties only after blocking $\mathrm{Cl}^{-}$-mediated inhibition, elevating local $\mathrm{K}^{+}$, or by applying sodium cyanide, so whether they are pacemakers during normal breathing, or contribute to the breathing rhythm, remains unresolved.

From our limited sample, GlyT2-EGFP preBötC pacemaker neurons express both $I_{\mathrm{NaP}}$ and $I_{\mathrm{CAN}}$. Whether GlyT2-EGFP preBötC pacemaker neurons have different biophysical, synaptic, or network properties from those of excitatory preBötC neurons also remains unresolved.

\section{Neurotransmitter phenotype of preBötC pacemaker neurons}

The hypothesis that pacemaker neurons play an obligatory role in respiratory rhythmogenesis in vitro arose from the observation that inhibition is not essential for generation of inspiratory rhythm (Feldman and Smith, 1989). The presence of inspiratorymodulated preBötC pacemaker neurons is well documented (Smith et al., 1991; Johnson et al., 1994; Thoby-Brisson and 
Ramirez, 2001; Del Negro et al., 2002; Peña et al., 2004), but in none of these papers (cf. St-John et al., 2009) was the transmitter phenotype determined. In the authors' interpretations of these various papers, and in models that cite them as evidence of the presence of pacemaker neurons [e.g., Butera et al. (1999a,b), Smith et al. (2000), Del Negro et al. (2001), and Rybak et al. $(2003,2008)]$, they are universally presumed to be excitatory. However, there is no a priori reason that pacemaker neurons need to be excitatory to either generate or modulate rhythm.

Models of respiratory rhythm in vitro recognize that inhibitory interactions are not obligatory as rhythm persists when synaptic inhibition is blocked. Nonetheless, such interactions could play a role in rhythmogenesis under normal conditions in more intact preparations, even in vivo, and certainly could affect patterning of respiratory output, even in slices. While inhibitory pacemaker neurons are obligatory for rhythmogenesis in other systems, such as in the generation of the pyloric rhythm in invertebrates (Cardi and Nagy, 1994; Mamiya and Nadim, 2004), we propose that the principal role of glycinergic preBötC pacemaker neurons is in modulation/stabilization of respiratory rhythm.

\section{Neuron size and sampling bias}

By virtue of exploiting visualized recording, we were limited to recording relatively superficial neurons in the slice, up to $\sim 120$ $\mu \mathrm{m}$ deep. However, patch-clamping methodologies used in several studies of preBötC pacemaker neurons can have a sampling bias toward neurons with larger somas with strong inspiratory drive. In the present sample, size was not an explicit or implicit criterion. There also is sampling bias in studies using blind patch recording (Johnson et al., 1994; Thoby-Brisson and Ramirez, 2001; Peña et al., 2004; St-John et al., 2009) and extracellular recording (Del Negro et al., 2001), which can record neurons much deeper in the slice but require detecting strong, likely somatic, electrical signals. This increases the probability of recording from larger somas, reducing the probability of recording smaller neurons that, according to our observations, are more likely to be glycinergic.

Establishing the role of any preBötC neuronal class, including pacemakers, in respiratory rhythm generation requires determination of their neurotransmitter phenotype. A basic requirement for validation of models that stipulate that excitatory pacemaker neurons are essential for rhythm generation in vitro is that this is actually the case. The demonstration of preBötC glycinergic pacemaker neurons suggests that this remains to be done.

\section{References}

Bianchi AL, Denavit-Saubié M, Champagnat J (1995) Central control of breathing in mammals: neuronal circuitry, membrane properties, and neurotransmitters. Physiol Rev 75:1-45.

Brockhaus J, Ballanyi K (1998) Synaptic inhibition in the isolated respiratory network of neonatal rats. Eur J Neurosci 10:3823-3839.

Büsselberg D, Bischoff AM, Paton JF, Richter DW (2001) Reorganisation of respiratory network activity after loss of glycinergic inhibition. Pflugers Arch 441:444-449.

Butera RJ Jr, Rinzel J, Smith JC (1999a) Models of respiratory rhythm generation in the pre-Bötzinger complex. I. Bursting pacemaker neurons. J Neurophysiol 82:382-397.

Butera RJ Jr, Rinzel J, Smith JC (1999b) Models of respiratory rhythm generation in the pre-Bötzinger complex. II. Populations of coupled pacemaker neurons. J Neurophysiol 82:398-415.

Cardi P, Nagy F (1994) A rhythmic modulatory gating system in the stomatogastric nervous system of Homarus gammarus. III. Rhythmic control of the pyloric CPG. J Neurophysiol 71:2503-2516.

Chitravanshi VC, Sapru HN (1996) NMDA as well as non-NMDA receptors mediate the neurotransmission of inspiratory drive to phrenic motoneurons in the adult rat. Brain Res 715:104-112.
Del Negro CA, Johnson SM, Butera RJ, Smith JC (2001) Models of respiratory rhythm generation in the pre-Bötzinger complex. III. Experimental tests of model predictions. J Neurophysiol 86:59-74.

Del Negro CA, Morgado-Valle C, Feldman JL (2002) Respiratory rhythm: an emergent network property? Neuron 34:821-830.

Del Negro CA, Morgado-Valle C, Hayes JA, Mackay DD, Pace RW, Crowder EA, Feldman JL (2005) Sodium and calcium current-mediated pacemaker neurons and respiratory rhythm generation. J Neurosci 25:446453.

Del Negro CA, Kam K, Hayes JA, Feldman JL (2009) Asymmetric control of inspiratory and expiratory phases by excitability in the respiratory network of neonatal mice in vitro. J Physiol 587:1217-1231.

Feldman JL, Del Negro CA (2006) Looking for inspiration: new perspectives on respiratory rhythm. Nat Rev Neurosci 7:232-242.

Feldman JL, Smith JC (1989) Cellular mechanisms underlying modulation of breathing pattern in mammals. Ann N Y Acad Sci 563:114-130.

Gray PA, Rekling JC, Bocchiaro CM, Feldman JL (1999) Modulation of respiratory frequency by peptidergic input to rhythmogenic neurons in the preBötzinger complex. Science 286:1566-1568.

Gray PA, Janczewski WA, Mellen N, McCrimmon DR, Feldman JL (2001) Normal breathing requires preBötzinger complex neurokinin-1 receptorexpressing neurons. Nat Neurosci 4:927-930.

Greer JJ, Smith JC, Feldman JL (1991) Role of excitatory amino acids in the generation and transmission of respiratory drive in neonatal rat. J Physiol 437:727-749.

Gutiérrez R, Heinemann U (2006) Co-existence of GABA and Glu in the hippocampal granule cells: implications for epilepsy. Curr Top Med Chem 6:975-978.

Guyenet PG, Sevigny CP, Weston MC, Stornetta RL (2002) Neurokinin-1 receptor-expressing cells of the ventral respiratory group are functionally heterogeneous and predominantly glutamatergic. J Neurosci 22:3806-3816.

Johnson SM, Smith JC, Funk GD, Feldman JL (1994) Pacemaker behavior of respiratory neurons in medullary slices from neonatal rat. J Neurophysiol 72:2598-2608.

Mamiya A, Nadim F (2004) Dynamic interaction of oscillatory neurons coupled with reciprocally inhibitory synapses acts to stabilize the rhythm period. J Neurosci 24:5140-5150.

Morgado-Valle C, Feldman JL (2007) NMDA receptors in preBötzinger complex neurons can drive respiratory rhythm independent of AMPA receptors. J Physiol 582:359-368.

Onimaru H, Arata A, Homma I (1990) Inhibitory synaptic inputs to the respiratory rhythm generator in the medulla isolated from newborn rats. Pflugers Arch 417:425-432.

Pagliardini S, Adachi T, Ren J, Funk GD, Greer JJ (2005) Fluorescent tagging of rhythmically active respiratory neurons within the pre-Bötzinger complex of rat medullary slice preparations. J Neurosci 25:2591-2596.

Peña F, Parkis MA, Tryba AK, Ramirez JM (2004) Differential contribution of pacemaker properties to the generation of respiratory rhythms during normoxia and hypoxia. Neuron 43:105-117.

Purvis LK, Smith JC, Koizumi H, Butera RJ (2007) Intrinsic bursters increase the robustness of rhythm generation in an excitatory network. J Neurophysiol 97:1515-1526.

Ramirez JM, Quellmalz UJ, Richter DW (1996) Postnatal changes in the mammalian respiratory network as revealed by the transverse brainstem slice of mice. J Physiol 491:799-812.

Rekling JC, Champagnat J, Denavit-Saubié M (1996) Electroresponsive properties and membrane potential trajectories of three types of inspiratory neurons in the newborn mouse brain stem in vitro. J Neurophysiol 75:795-810.

Ren J, Greer JJ (2006) Modulation of respiratory rhythmogenesis by chloride-mediated conductances during the perinatal period. J Neurosci 26:3721-3730.

Richter DW, Spyer KM (2001) Studying rhythmogenesis of breathing: comparison of in vivo and in vitro models. Trends Neurosci 24:464-472.

Ruangkittisakul A, Schwarzacher SW, Secchia L, Poon BY, Ma Y, Funk GD, Ballanyi K (2006) High sensitivity to neuromodulator-activated signaling pathways at physiological $\left[\mathrm{K}^{+}\right]$of confocally imaged respiratory center neurons in on-line-calibrated newborn rat brainstem slices. J Neurosci 26:11870-11880.

Rybak IA, Shevtsova NA, St-John WM, Paton JF, Pierrefiche O (2003) En- 
dogenous rhythm generation in the pre-Bötzinger complex and ionic currents: modelling and in vitro studies. Eur J Neurosci 18:239-257.

Rybak IA, O'Connor R, Ross A, Shevtsova NA, Nuding SC, Segers LS, Shannon R, Dick TE, Dunin-Barkowski WL, Orem JM, Solomon IC, Morris KF, Lindsey BG (2008) Reconfiguration of the pontomedullary respiratory network: a computational modeling study with coordinated in vivo experiments. J Neurophysiol 100:1770-1799.

Shao XM, Feldman JL (1997) Respiratory rhythm generation and synaptic inhibition of expiratory neurons in pre-Bötzinger complex: differential roles of glycinergic and GABAergic neural transmission. J Neurophysiol 77:1853-1860.

Simat M, Ambrosetti L, Lardi-Studler B, Fritschy JM (2007) GABAergic synaptogenesis marks the onset of differentiation of basket and stellate cells in mouse cerebellum. Eur J Neurosci 26:2239-2256.

Smith JC, Ellenberger HH, Ballanyi K, Richter DW, Feldman JL (1991) PreBötzinger complex: a brainstem region that may generate respiratory rhythm in mammals. Science 254:726-729.

Smith JC, Butera RJ, Koshiya N, Del Negro C, Wilson CG, Johnson SM (2000) Respiratory rhythm generation in neonatal and adult mammals: the hybrid pacemaker-network model. Respir Physiol 122:131-147.

St-John WM, Stornetta RL, Guyenet PG, Paton JF (2009) Location and properties of respiratory neurones with putative intrinsic bursting properties in the rat in situ. J Physiol 587:3175-3188.
Stornetta RL, Rosin DL, Wang H, Sevigny CP, Weston MC, Guyenet PG (2003) A group of glutamatergic interneurons expressing high levels of both neurokinin- 1 receptors and somatostatin identifies the region of the pre-Bötzinger complex. J Comp Neurol 455:499-512.

Tan W, Janczewski WA, Yang P, Shao XM, Callaway EM, Feldman JL (2008) Silencing preBötzinger complex somatostatin-expressing neurons induces persistent apnea in awake rat. Nat Neurosci 11:538-540.

Thoby-Brisson M, Ramirez JM (2001) Identification of two types of inspiratory pacemaker neurons in the isolated respiratory neural network of mice. J Neurophysiol 86:104-112.

Wang H, Stornetta RL, Rosin DL, Guyenet PG (2001) Neurokinin-1 receptor-immunoreactive neurons of the ventral respiratory group in the rat. J Comp Neurol 434:128-146.

Winter SM, Fresemann J, Schnell C, Oku Y, Hirrlinger J, Hülsmann S (2009) Glycinergic interneurons are functionally integrated into the inspiratory network of mouse medullary slices. Pflugers Arch 458:459-469.

Zeilhofer HU, Studler B, Arabadzisz D, Schweizer C, Ahmadi S, Layh B, Bösl MR, Fritschy JM (2005) Glycinergic neurons expressing enhanced green fluorescent protein in bacterial artificial chromosome transgenic mice. J Comp Neurol 482:123-141.

Zhang W, Elsen F, Barnbrock A, Richter DW (1999) Postnatal development of GABAB receptor-mediated modulation of voltage-activated $\mathrm{Ca} 2+$ currents in mouse brain-stem neurons. Eur J Neurosci 11:2332-2342. 